\title{
Proteome-wide evidence for enhanced positive Darwinian selection within intrinsically disordered regions in proteins
}

\author{
Johan Nilsson ${ }^{1}$, Mats Grahn ${ }^{1}$ and Anthony PH Wright ${ }^{1,2^{*}}$
}

\begin{abstract}
Background: Understanding the adaptive changes that alter the function of proteins during evolution is an important question for biology and medicine. The increasing number of completely sequenced genomes from closely related organisms, as well as individuals within species, facilitates systematic detection of recent selection events by means of comparative genomics.

Results: We have used genome-wide strain-specific single nucleotide polymorphism data from 64 strains of budding yeast (Saccharomyces cerevisiae or Saccharomyces paradoxus) to determine whether adaptive positive selection is correlated with protein regions showing propensity for different classes of structure conformation. Data from phylogenetic and population genetic analysis of 3,746 gene alignments consistently shows a significantly higher degree of positive Darwinian selection in intrinsically disordered regions of proteins compared to regions of alpha helix, beta sheet or tertiary structure. Evidence of positive selection is significantly enriched in classes of proteins whose functions and molecular mechanisms can be coupled to adaptive processes and these classes tend to have a higher average content of intrinsically unstructured protein regions.

Conclusions: We suggest that intrinsically disordered protein regions may be important for the production and maintenance of genetic variation with adaptive potential and that they may thus be of central significance for the evolvability of the organism or cell in which they occur.
\end{abstract}

\section{Background}

Understanding the process of adaptation is of central importance for many biological questions, such as how species respond to climate changes, pathogens or other environmental perturbations, as well for the mechanisms underlying genetic diseases, such as cancer. Evolutionary adaptation occurs when an inheritable change in the phenotype of an organism makes it more suited to its present environment. In diseases like cancer, adaptive mutations allow individual cells within multi-cellular organisms to thrive at the expense of neighbouring cells by over-riding the normal cellular controls that restrict cell growth and division. At the molecular level such phenotypic changes are the result of mutational processes acting on either protein-coding or non-coding DNA sequences. Although the neutral theory of evolution [1] predicts the vast

\footnotetext{
* Correspondence: anthony.wright@ki.se

'School of Life Sciences, Södertörn University, SE-141 89 Huddinge, Sweden Full list of author information is available at the end of the article
}

majority of mutations to be either deleterious or neutral, recent years have seen a sharp increase in publications indentifying the action of positive Darwinian selection on genes in various species [2]. The rapidly increasing number of completely sequenced genomes, along with improved bioinformatic methodologies for detecting evidence of selection [3-5], has enabled large-scale scanning of genes or genetic elements for evidence of positive selection. In particular, comparative approaches using sets of genomes from closely related species, or strains within a species, have proven powerful in detecting genes or genetic regions under recent positive selection [6-8].

SNPs are the most abundant source of genetic variation affecting populations. SNPs found within a protein-coding region may be classified as synonymous SNPs or nonsynonymous SNPs, depending on whether the encoded amino acid is altered in the alternative DNA sequence variants. Non-synonymous SNPs in coding sequences, together with SNPs in gene regulatory regions, are

C Biomed Central

(c) 2011 Nilsson et al.; licensee BioMed Central Ltd. This is an open access article distributed under the terms of the Creative Commons Attribution License (http://creativecommons.org/licenses/by/2.0), which permits unrestricted use, distribution, and reproduction in any medium, provided the original work is properly cited. 
believed to have the highest impact on phenotype [9] and hence they are suitable targets for studies on adaptation. However, a major task is still to understand which of the 10 million or so SNPs in the human genome are of functional significance. There is therefore a need for approaches that help to predict the subclass of SNPs that are more likely to be of adaptive significance. The relevance of this task is underscored by the International HapMap Project, which uses genetic variation as a tool to better understand the molecular basis of human disease as well as the mechanisms underlying pharmaceutical therapy [10].

Evolvability is often described as an organism's capacity to generate heritable phenotypic variation [11-13]. This capacity may either entail a reduction in the potential lethality of mutations or a reduction in the number of mutations required to generate phenotypically novel traits [14-17]. At the molecular level, non-synonymous SNPs in a protein-coding gene may result in structural changes in the encoded protein, which may cause phenotypic changes and an increased potential for evolutionary innovation, either directly or in future environments [15]. Proteins consist of conformationally structured regions, containing $\alpha$-helices and $\beta$-sheets, as well as intrinsically disordered regions that are conformationally flexible. Intrinsically disordered protein regions (IDRs) have been a recent focus of attention [18-21]. IDRs are abundant in the eukaryotic proteome, with an estimated 50 to $60 \%$ of all Saccharomyces cerevisiae proteins containing at least one disordered segment comprising more than 30 amino acid residues [22]. Interestingly, IDRs occur more frequently in eukaryotes than in bacteria or archea, perhaps suggesting a role in the evolution of eukaryotes [23]. To our knowledge, the relationship between recent adaptation and the different types of structural domains within proteins has not been systematically studied.

The budding yeast $S$. cerevisiae is one of the best-studied model organisms at the molecular level. It was the first eukaryotic genome to be fully sequenced [24], and it has a well-annotated proteome [25]. The relatively small sizes of fungal genomes, along with recent advances in whole genome sequencing, have facilitated the establishment of multiple yeast genome sequences [26-29]. From an evolutionary perspective, the short generation time of yeasts combined with the strong environmental selective pressures to which they are exposed facilitate the detection of recent selection events in these organisms. Indeed, different budding yeast species display a surprisingly high level of genome diversity that is comparable to that observed within the family of chordates [27]. The Saccharomyces Genome Resequencing Project has resulted in genomic sequences of multiple strains of $S$. cerevisiae and its close relative, Saccharomyces paradoxus [30]. Studying polymorphism and divergence between the genomes of
S. cerevisiae and S. paradoxus strains thus provides an excellent opportunity to identify genes or genetic regions likely to be under positive Darwinian selection.

In this study, we performed genome-wide analyses of SNPs identified in the Saccharomyces Genome Resequencing Project that lie within protein coding genes and used phylogenetic and population genetic methods to detect evidence of selection acting either on entire protein-coding genes or on individual codon sites within genes. Interestingly, we found a stronger association of both genes and codons under positive selection with intrinsically disordered protein regions compared to regions of regular secondary or tertiary structure. Furthermore, a higher degree of positive selection was found to act on proteins belonging to different functional and structural protein categories that are characterized by a high average IDR content. The biological significance of these findings is discussed in the context of the structure, function and evolvability of proteins.

\section{Results}

The frequency of codon sites under positive selection is enhanced in protein regions with intrinsically disordered structure

The Fixed Effects Likelihood (FEL) method was used to predict codon sites under selection in the coding regions of 3,746 S. cerevisiae protein coding genes, for which inter-species alignments could be reliably constructed and for which no recombination events were predicted in the 37 S. cerevisiae and 27 S. paradoxus genome sequences used (Figure 1). One or more codon sites were predicted to be under selection in 3,421 of these genes. As expected, the total number of sites predicted to be under positive selection (7,561 sites) was considerably lower than the number of sites predicted to be under negative selection (178,408 sites).

To investigate whether the pattern of selection on individual codon sites is correlated with the structural context of the encoded amino acids, the frequency of positively and negatively selected sites in IDRs as well as structured regions ( $\alpha$-helices and $\beta$-strands) was compared. Regions of regular secondary structure and IDRs were predicted using PSIPRED and VSL2, respectively. Frequency differences were assessed by a $\chi^{2}$ test. The ratio of positive to negative sites was approximately three-fold higher in IDRs compared to regions of regular secondary structure, for which the ratio was similar in $\alpha$ helical and $\beta$-strand regions (Figure 2a). To investigate whether the higher ratio of positive to negative sites in IDRs was mainly due to an excess of positive sites or a depletion of negative sites, the mean proportion of positively and negatively selected codon sites in the three structural conformation states was investigated. Interestingly, the proportion of negatively selected sites was not 


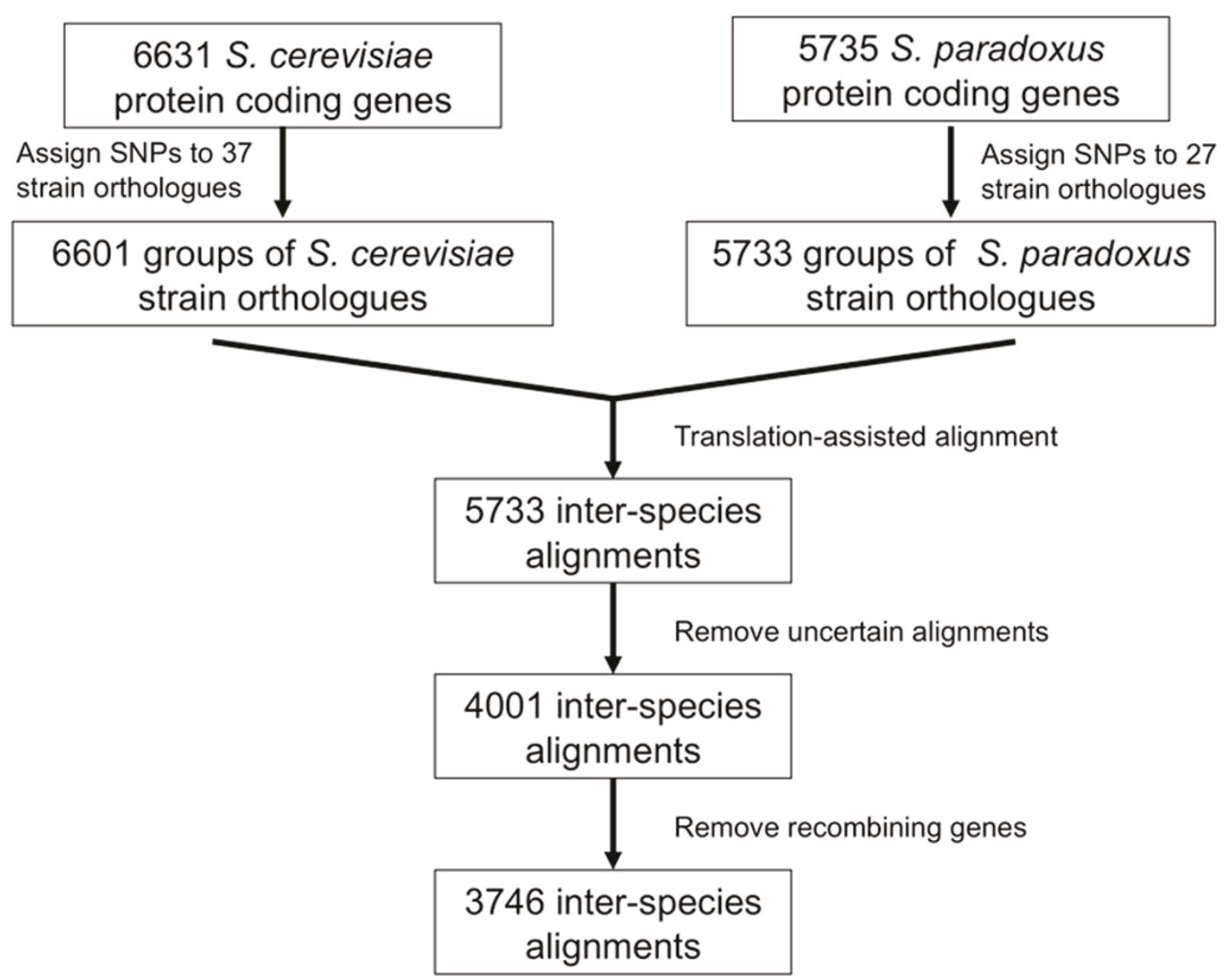

Figure 1 Flow chart illustrating the initial processing of the source data. The diagram show the steps involved in creating multiple alignments including S. cerevisiae and S. paradoxus strains as well as the number of genes involved at each step. Filtering steps for removal of uncertain alignments are also shown. See Materials and methods for details.

significantly lower in IDRs compared to regions of regular secondary structure, whereas the proportion of positively selected sites was almost threefold higher in IDRs (Figure 2b). We thus conclude that there was a strong enrichment of positively selected sites in IDRs compared to regions of regular secondary structure, whereas the distribution of negatively selected sites was similar in regions of structured and disordered conformation. Simulation experiments have suggested that selective forces might act more strongly on longer IDRs $(\geq 30$ amino acid residues) compared to shorter disordered sequences or secondary structure elements [31]. Further, it has been suggested that selective forces affecting long IDRs might be similar to those affecting the tertiary structure domains of proteins [32]. We therefore calculated the ratio of predicted positive to negative codon sites in tertiary structure domains and IDRs that were 30 or more residues in length. Figure $2 \mathrm{c}$ shows that the relative frequency of positive selection in long IDRs is greater that in regions of tertiary structure. This is due to an elevated frequency of positively selected codons in the long IDRs.

To independently test whether the observed frequency differences were greater than would be expected by chance, a randomization test was performed. Briefly, the test entailed sampling a number of selected sites, equivalent to the number of sites found for each of the three conformational states individually, from the combined set of selected sites. The number of sites under either positive or negative selection in each such sample was then calculated. The procedure was repeated 10,000 times to obtain an empirical distribution of the number of selected sites expected by chance. The null hypothesis that the actual number of sites under selection for each conformational state belonged to the derived distributions of selected sites was assessed by a $t$-test. The results 
(a)

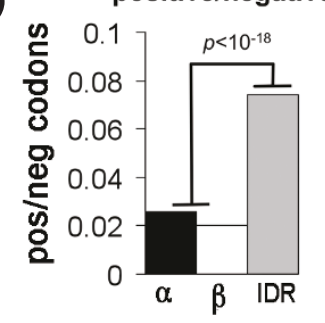

(b)

negative

positive

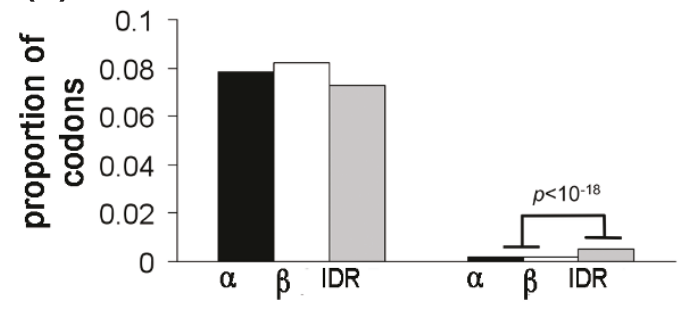

(c) positive/negative

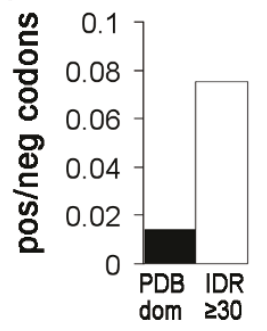

(d)

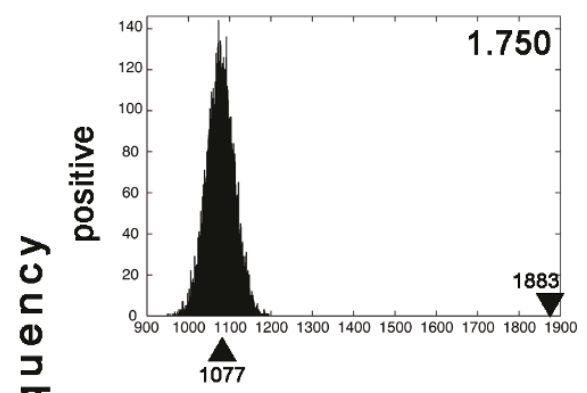

这

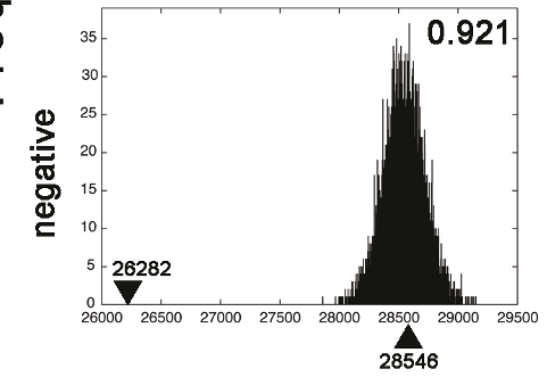

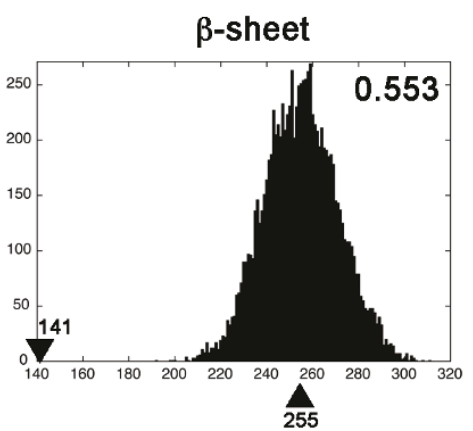

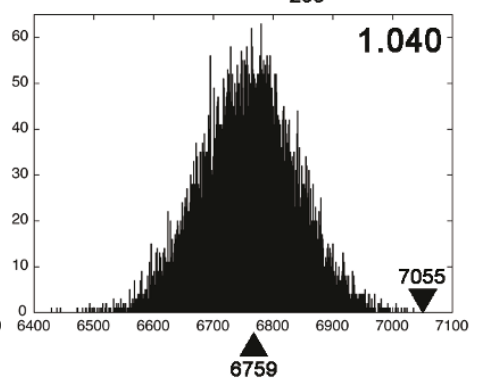

$\alpha$-helix
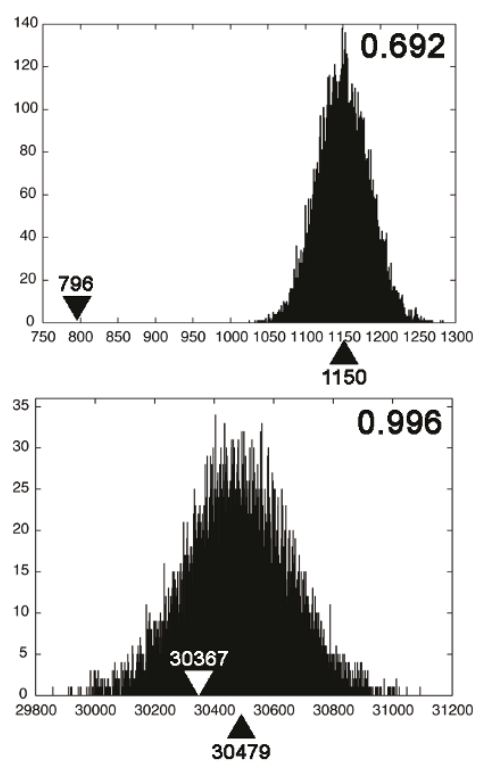

Number of residues under selection

Figure 2 Codon sites under positive selection are over-represented in gene regions encoding intrinsically disordered regions of proteins. (a) The ratio of positive to negative sites is higher in IDRs than in regions of regular protein structure. The ratio of positive to negative sites is shown for protein regions predicted to have $\alpha$-helical $(\alpha), \beta$-sheet $(\beta)$ or intrinsically disordered (IDR) protein conformation. The $P$-value shows the significance of the difference between the ratio associated with IDRs in relation to regions of regular structure (a $\chi^{2}$ test was used to test the null hypothesis that there is no difference between the ratios associated with different protein conformation classes). (b) The proportion of codons under selection is enhanced in IDRs for positively selected sites but not negatively selected sites. Annotations are as for (a).

Differences between the frequencies of negative sites in regions of different protein conformation were not significant. (c) The ratio of positive to negative sites is higher in long IDRs than in structured protein domains. The ratio of positive to negative sites is shown for protein regions within known protein domains (PDB dom) or predicted intrinsically disordered protein regions of at least 30 residues in length (IDR $\geq 30$ ). The frequency of positively selected codons in IDR $\geq 30$ and PDB dom is 0.0055 and 0.0011 , respectively, while the equivalent frequencies for negatively selected codons are 0.0728 and 0.0750 , respectively. (d) Codons under positive selection are significantly more frequent in IDRs than expected in relation to an empirically generated random distribution of selected sites. The panels show empirical frequency distributions (histograms) predicted for a random distribution of positively and negatively selected sites within protein regions with intrinsically disordered structure (IDR), $\beta$-sheet and $\alpha$-helix conformation, generated by 10,000 randomization trials. The median of each distribution is shown associated with upward-pointing arrowheads and the observed number of selected sites together with downward-pointing arrowheads. The ratio of the observed number of sites in relation to the median of the random distribution is shown in the upper right corner of each panel. The ratio is significantly different from unity in all cases $\left(P \leq 10^{-3}\right)$ except for negative sites in $\alpha$-helical regions.

showed a significant $(P \leq 0.001)$ difference between the observed frequencies of selected sites in different conformational states and the empirically generated random distributions in all cases except in the case of negatively selected sites in $\alpha$-helical regions. Figure 2d (left panel) shows the derived distributions from each randomization test along with the observed number of positively and negatively selected sites (downward-pointing arrowheads) for IDRs. The figure provides independent support for a strong enrichment of positively selected sites in IDRs and 
a small but significant depletion of negatively selected sites in these regions. The relative difference between the number of observed (downward-pointing arrowheads) and expected (upward-pointing arrowheads) sites under selection was much greater for positively than for negatively selected sites, as shown by the ratio of the two values (top right corner in each panel). The enrichment level for positively selected sites in IDRs is almost ten-fold higher than the under-representation level of negatively selected sites in the same regions. Hence, the distribution was considerably less skewed for negatively selected sites. The trend was exactly the opposite for regions with $\alpha$-helical (right panels) and $\beta$-sheet (middle panels) conformation. Positively selected sites are underrepresented in these regions. Again the extent of positive site under-representation is much greater than the deviation level for negative sites, which differ little, if at all, from the empirically generated value expected for a random distribution within the $\alpha$-helical and $\beta$-sheet conformational classes. Based on the proteome-wide analysis of codons under selection, we thus concluded that there is a strong bias in the distribution of positively selected sites between gene regions encoding regular and disordered protein structure.

We next investigated whether a similar bias in the distribution of codons under selection could be observed at the level of intact genes. To this end, a non-overlapping sliding window of 25 codons was moved across each aligned gene in the analyzed data set, and the number of positively selected codon sites within each window was counted. The predicted IDR content within each window was also calculated. Each window containing at least one positive site thus generated a data point and for genes resulting in at least five such data points the correlation between IDR content and the number of codons under positive selection was assessed by calculation of Spearman's rank correlation coefficient $(P \leq$ 0.05). Again, the correlation between degree of disorder and incidence of positive selection was obvious. For the genes analyzed, a significant positive correlation between IDR content and positively selected codon sites was observed in 528 genes, whereas a significant negative correlation was found in only 28 genes. These results thus suggest that the correlation between positively selected sites and gene regions encoding IDRs can be extended to the level of intact genes and proteins.

\section{Intrinsically disordered protein regions have a higher proportion of fixed non-synonymous polymorphisms} Having observed that intrinsically disordered protein regions were enriched in codon sites under positive selection, we next used an alternative approach to investigate whether enhanced positive selection in genes with high IDR content could be observed at the level of intact genes. The McDonald-Kreitman test was used to estimate the degree of selection acting on the 3,746 aligned S. cerevisiae and S. paradoxus protein coding genes by means of the fixation index (FI; see Materials and methods for details). Similar to the codon level, a minority of genes were predicted to be under positive selection (FI > 1; 128 genes under a $P$-value threshold of 0.05 ), while a larger number were predicted to be under negative selection (FI $<1 ; 519$ genes under a $P$-value threshold of $0.05)$. Figure $3 a$ shows the FI as a function of IDR content for each of the analyzed genes and the equivalent plot for regular secondary structure regions is shown in Figure 3b. Spearman's rank correlation coefficient was calculated to assess the correlation between secondary structure content and FI values, and a $t$-test was used to determine its statistical significance. Consistent with our results at the individual codon level, there was a significant $\left(P \leq 10^{-18}\right)$ tendency for FI and IDR content to be correlated $\left(r_{s}=0.28\right)$. A negative correlation of similar magnitude was seen between FI and regular secondary structure content $\left(r_{s}=-0.26, P \leq 10^{-18}\right)$. As a negative control, we similarly assessed the level of correlation between $(\mathrm{G}+\mathrm{C})$ content and FI (Figure 3c), and between $(\mathrm{G}+\mathrm{C})$ content and IDR content (Figure 3d). No significant correlation was found with $r_{s}$ values of 0.01 for correlation of $(\mathrm{G}+\mathrm{C})$ content with both FI and IDR content. Removal of 63 outliers (genes with a fixation index deviating more than three standard deviations from the mean of the entire data set) did not significantly affect any of the obtained results (data not shown).

A Mann-Whitney $U$ test was also performed in order to independently test the significance of the correlation between FI values and IDR content. Genes were sorted into two equally sized groups according to the level of their FI value (the median FI value was 0.42 after removal of outliers). The null hypothesis of equal secondary structure content in the resulting data sets was then tested. There was a significantly higher IDR content in the dataset containing higher FI values $\left(P \leq 10^{-}\right.$ $\left.{ }^{15}\right)$. No significant difference in FI or IDR content $(P>$ $0.5)$ was found between subsets when the dataset was divided in the same way into subsets of high and low (G $+C$ ) content (the median $G+C$ value was 0.42 ). Thus, we conclude that there is a higher proportion of fixed nonsynonymous polymorphism in IDRs than in other protein regions, again suggesting an enhanced level of positive selection in these regions.

A potential problem with the analyses presented above is the fact that most genes did not obtain a statistically significant FI value at the chosen level of significance, and hence were discarded from the analysis. To assure that this did not prejudice the overall conclusion, we performed an alternative, proteome-wide analysis. Three composite alignments were created by concatenating 


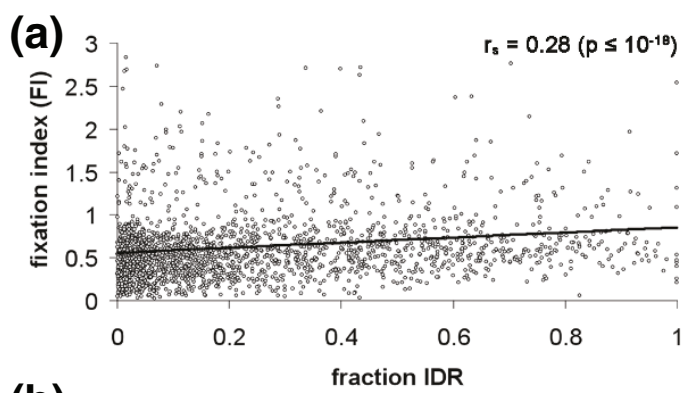

(b)

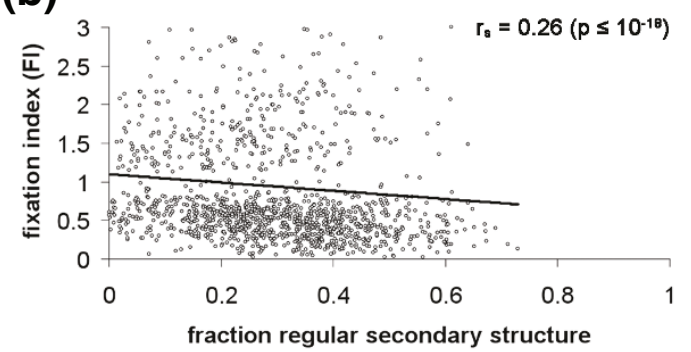

(c)

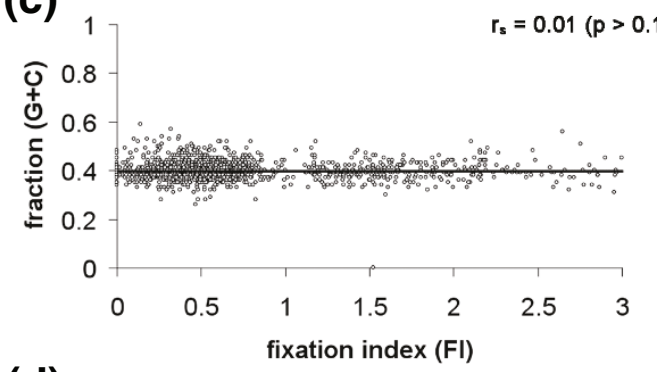

(d)

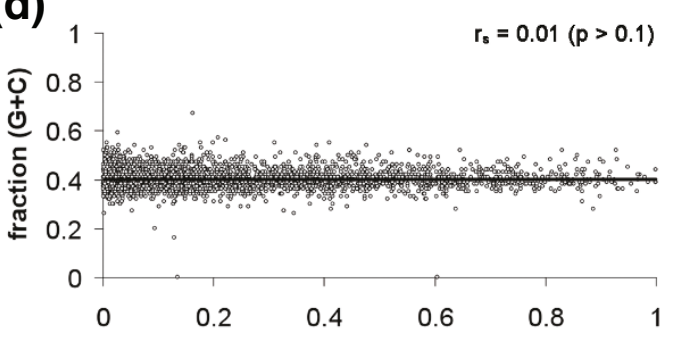

(e)

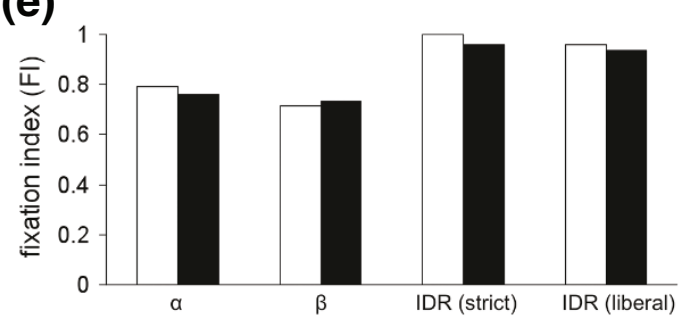

Figure 3 Relative levels of species-specific fixation of variant SNP alleles in each gene are correlated with the level of intrinsically disordered region content in the corresponding proteins. $(\mathbf{a}, \mathbf{b})$ Scatter plot showing the fixation index (FI) for genes, calculated by the McDonald-Kreitman test (see Materials and methods), is positively correlated with the fraction of IDR (a) and negatively correlated with the fraction of regular secondary structure $(b)$ in the corresponding proteins. Spearman's rank correlation coefficients $\left(r_{s}\right)$ and associated $P$-values are shown. (c, d) The $(\mathrm{G}+\mathrm{C})$ content of genes is not correlated with their Fl (c) or with the fraction of IDR in the corresponding proteins (d). Spearman's rank correlation coefficients $\left(r_{s}\right)$ and associated $P$-values are shown. (e) The mean Fl corresponding to all IDRs studied is higher than that for all $\alpha$-helical regions or $\beta$-sheet regions studied. The FI for concatenated tracts of predicted $\alpha$-helical $(\alpha), \beta$-sheet $(\beta)$ and IDRs are plotted. Values are shown for IDR predictions using confidence thresholds of 0.8 (strict) or 0.5 (liberal) (see Materials and methods for details). Open bars designate results obtained for the non-filtered data set while the filled bars designate the data set after removal of outliers (see Materials and methods for details). 
protein regions from all 3,746 aligned genes that are predicted to be $\alpha$-helix, $\beta$-strand or IDR. The overall FI was then calculated for each of the three concatenated alignments. Figure 3e shows the resulting overall FI for each composite alignment. In accordance with our previous observations, the overall FI value was close to 1.0 in the IDRs, indicating an overall balance between positive and negative selection acting within these regions. These results were very similar whether a strict or a liberal confidence value was used in the IDR predictions (see Materials and methods). In protein regions with regular secondary structure, the overall FI value was lower than 1.0, indicating an overall bias towards purifying selection acting on these regions. Thus, the data support enhanced positive selection in IDRs even when data from all the gene alignments are studied.

Finally, as an independent assessment of the distribution bias of positively selected polymorphic sites within genes, a non-overlapping window of 25 codons was moved over all the gene alignments, and a regional FI was calculated within each such window. The correlation between the resulting FI and IDR content was estimated by Spearman's rank correlation coefficient. The number of genes with a positive correlation between intrinsic disorder and FI (329 genes) was about an order of magnitude higher than the number of genes where a negative correlation was observed (39 genes), again suggesting a positive correlation between intrinsic disorder and degree of positive selection within proteins.

\section{Intrinsically disordered regions are not depleted in functional sites}

Given the higher frequency of positively selected amino acid-altering substitutions observed in IDRs, we wanted to further exclude the possibility that this was merely a consequence of a lower level of functional sites in these regions. To this end, we compared the distribution of predicted functional sites between IDRs and non-IDRs using the Limacs functional sites index, for which values show the ratio of functional sites in IDRs in relation to their level in non-IDRs (see Materials and methods). Although we might have expected most annotated functional domains studied by this method to consist mainly of regular secondary structure elements, previous studies have shown that conserved disordered regions occur frequently in annotated protein domains [33]. The mean IDR content in mapped Pfam domains was shown to be about $26 \%$, using a confidence value threshold of 0.5 for IDR prediction (compared to a content of about $44 \%$ for the entire proteome). Using a more stringent confidence value threshold (0.8) the equivalent values for IDR content were $7.4 \%$ and $26 \%$, respectively. As shown in Figure 4, the Limacs functional sites index was close to or in excess of 1.0 for most IDR prediction parameter

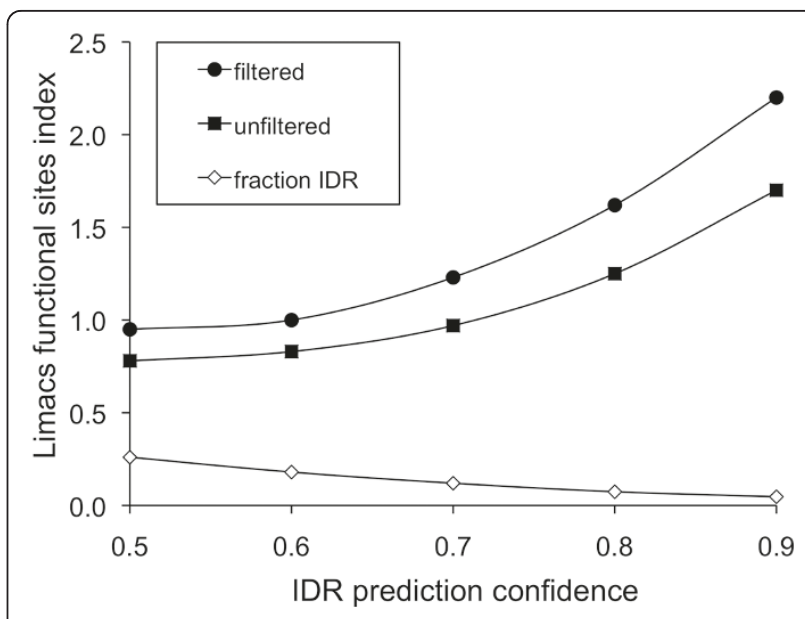

Figure 4 Functional amino acid residues are not underrepresented in intrinsically disordered regions within proteins. The Limacs functional sites index calculated for mapped Pfam domains within IDRs is plotted against different confidence value thresholds used for prediction of IDRs. The mean fraction of residues predicted to be in IDRs relative to structured regions, at different prediction threshold values, is indicated by open diamonds (default threshold used in the study was 0.8). The corresponding Limacs functional sites index is shown without filtering (filled

squares) or after filtering to remove multiple examples of the same Pfam domain (filled circles; see Materials and methods for details).

settings, suggesting that functional sites are at least as frequent in IDRs as they are in non-IDRs. Somewhat higher relative levels of functional sites were detected in IDRs after filtering the IDR and non-IDR data sets by removing duplicate examples of Pfam domains that occur in two or more proteins in order to prevent possible bias from Pfam domains that are found in many proteins. The Limacs functional sites index increases for both the filtered and non-filtered data sets as the stringency for IDR prediction is increased. Thus, the high relative identification of Limacs sites in IDRs cannot be accounted for by their preferential occurrence in falsely identified IDRs at low stringency levels. Taken together with the relatively high level of negatively selected codons in IDRs and the relatively high FI for polymorphisms in IDRs, these data provide independent evidence that the high levels of apparent adaptive genetic variation predicted for IDRs is not a consequence of reduced negative selection acting on amino acid residues located in IDRs.

\section{Positively selected sites are over-represented in a subset of functional protein categories}

To determine the generality of enhanced positive selection in IDRs, we next wanted to investigate how codon sites under positive and negative selection are distributed between different functional classes of proteins. To this end, we used two alternative protein annotation 
schemes from the Munich Information Center for Protein Sequences (MIPS), FunCat and ProteinCat [34]. A randomization test was employed to detect whether a statistically significant excess of selected sites occurred in any of the subcategories in either catalogue. Figure 5 shows categories significantly enriched in positively (filled bars) or negatively (open bars) selected residues, using a $P$-value threshold of 0.01. In FunCat (Figure 5a), statistical support for positively selected residues is found in proteins involved in both cell growth and morphogenesis, including mating, cell signaling, virulence and defense, as well as various aspects of nucleic acid biology, including the replication, repair, recombination and transcription of DNA. Enrichment of negatively selected residues was observed for a smaller number of categories, including conserved metabolic processes, such as fermentation and detoxification, as well as for protein folding and stabilization. In ProteinCat (Figure $5 \mathrm{~b})$, fewer categories were enriched in positively selected sites but all are associated with transcription factors. Most categories are enriched in negatively selected residues and mainly represent different categories of enzymes. The clearest common conclusion from analysis of both catalogues is that transcription factors tend to be enriched in positively selected amino acid residues.

\section{Protein categories with a high propensity for positive selection have a high average IDR content}

Given the correlation between positive selection and both the IDR content of proteins and their functional categorization, we were interested to test directly whether the average IDR content of protein categories is generally correlated with their content of positively or negatively selected sites. To investigate this, the major categories in FunCat and ProteinCat were sorted into ranks according to their average IDR content (Figure 6). The ranks of values for FunCat (Figure 6a) and ProteinCat (Figure 6b) categories show clearly that categories enriched in positively selected sites (filled squares) tend to have higher average IDR contents while the reverse is true for categories enriched in negatively selected sites (open triangles). Transcription factor categories that are significantly enriched in positively selected sites lie closest to the top of both category ranks. We conclude that transcription factors may provide good examples of proteins in which IDRs play an important role in functional adaptation.

\section{Discussion}

Here we show evidence for association between positive adaptive selection and regions of proteins with a low intrinsic propensity for secondary structure formation. This conclusion is based on the study of how genetic variation within 64 strains of $S$. cerevisiae and $S$. paradoxus affects the amino acid sequence of about two-thirds of the proteins within the yeast proteome. Since we cannot reconstruct the evolutionary history of these strains, it is relevant to discuss issues that influence the robustness of our conclusions.

Firstly, we have addressed whether the conclusions we draw could be influenced by the selection of gene alignments for study since we have not studied all genes. Genes were mainly excluded from the study based on uncertainty of the alignments. For the analysis shown, we required a level of $70 \%$ amino acid identity in proteins translated from the aligned genes. Reducing this threshold to $60 \%$ did not increase the number of proteins appreciably, probably because many of the low quality alignments result from incomplete genome sequences for one or more of the strains. An increase of the threshold to $80 \%$ identity, however, led to the exclusion of a further 800 gene alignments. Importantly, the use of these different thresholds for selection of gene alignments for study did not significantly influence the conclusions drawn.

Secondly, we have used different approaches to identify evidence of natural selection since each individual method may be subject to potential drawbacks. While the accuracy of maximum likelihood methods for identifying codons under selection has been questioned recently $[35,36]$, the McDonald-Kreitman approach is an insensitive method for detecting positive selection because evidence of positive selection is often cancelled out by negative selection, which is much more common. Indeed, the recent study by Liti et al. [30] did not find any statistical support for the existence of individual genes under positive selection when McDonald-Kreitman data were corrected for random effects associated with multiple testing. We have not corrected the data in our analysis since the aim was to study the overall association of protein structure with propensity for positive or negative selection rather than to identify individual genes under selection. The fact that we identify evidence for similar patterns of positive and negative selection at the level of codons using the FEL method and at the level of intact genes or gene regions using the McDonald-Kreitman test strongly supports the conclusion that the propensity for positive selection is enhanced in the IDRs of proteins. Nowaza et al. [36] have pointed to the utility of correlating bioinformatic predictions of codon sites under positive selection with biochemical data. Our observation that predicted evidence of positive selection tends to correlate with IDRs in proteins will be a useful parameter to test in other systems.

Thirdly, we have used several alternative strategies and statistical tests, including permutation tests of empirical significance levels, to assess the significance of the associations we have observed in the different tests for 


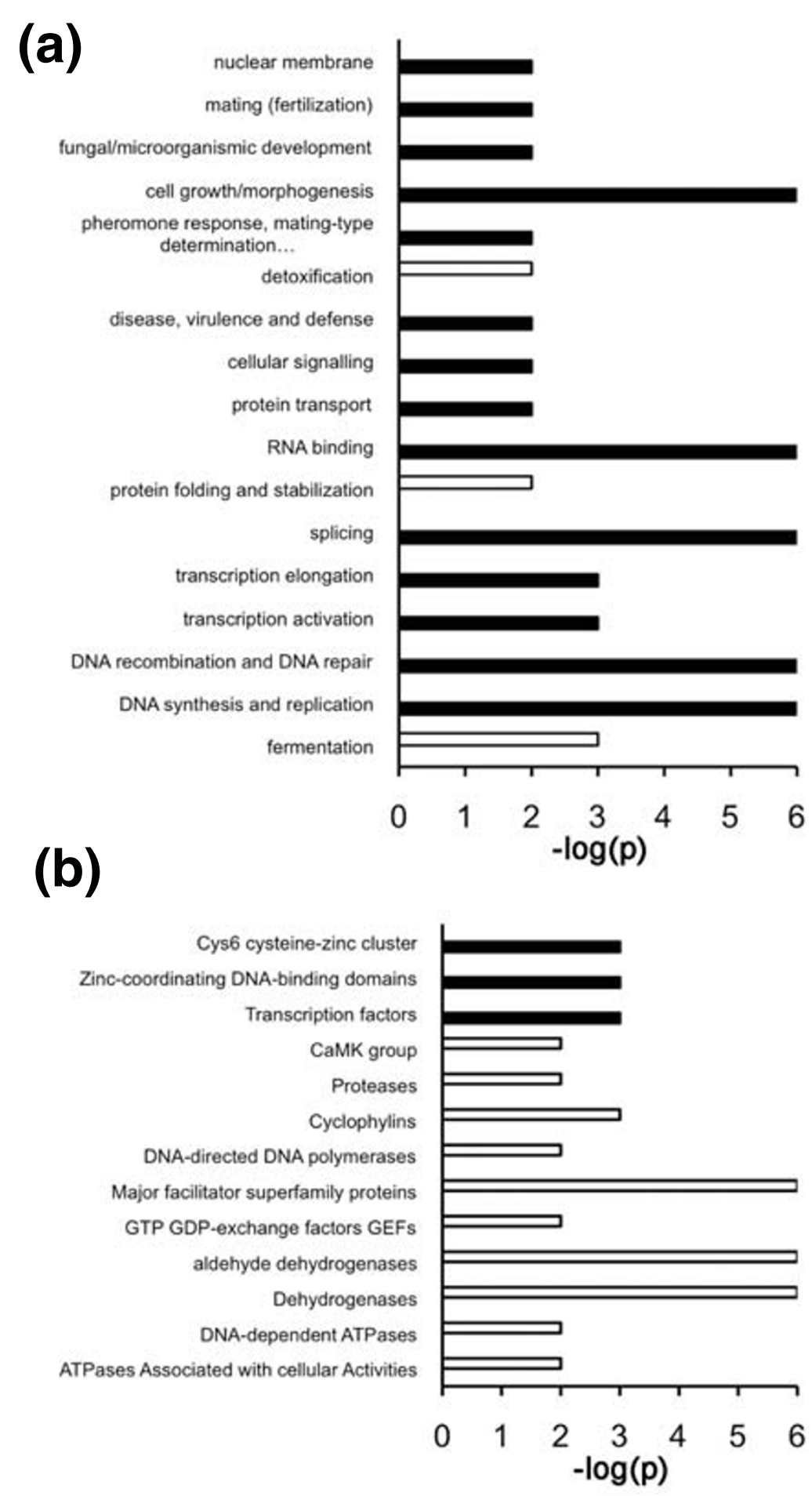

Figure 5 Specific protein categories are significantly over-represented in their content of codon sites under positive or negative selection. (a) Functional categories of the MIPS FunCat proteins that show significant $(P \leq 0.01)$ enrichment of codon sites under positive (filled bars) or negative (open bars) selection. (b) Functional categories of the MIPS ProteinCat proteins that show significant $(P \leq 0.01)$ enrichment of codon sites under positive (filled bars) or negative (open bars) selection. 
(a)

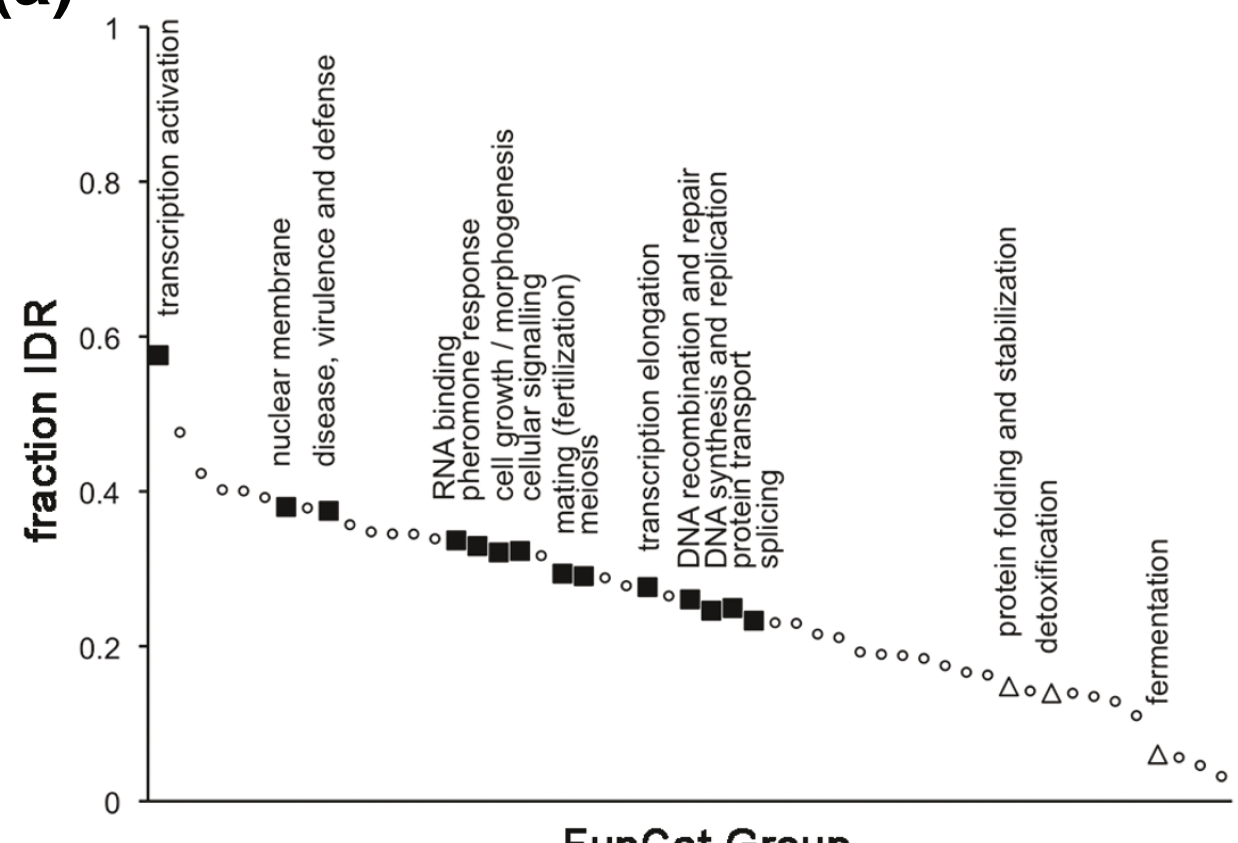

FunCat Group

(b)

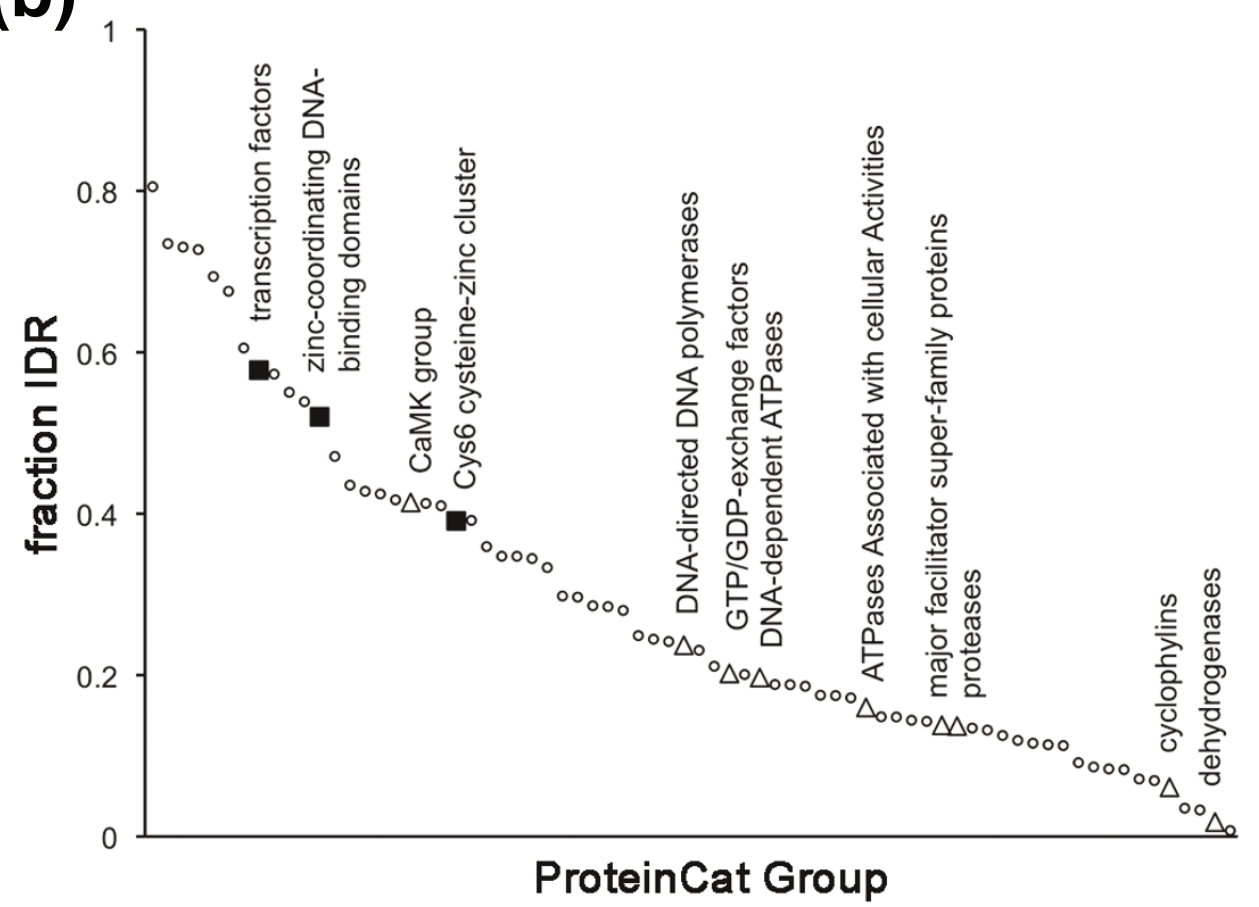

Figure 6 Protein categories enriched in codon sites under positive selection tend to have higher average levels of intrinsically disordered regions compared to categories enriched in sites under negative selection. (a) MIPS FunCat categories are plotted in a rank according to their IDR content (small open circles). Categories from Figure 5a that are enriched in codon sites under positive (filled squares) or negative (open triangles) selection are plotted with a larger symbol. (b) MIPS ProteinCat classes, including those enriched in codon sites under selection (Figure 5b), are plotted as in (a). 
positive and negative selection. In all cases these tests provide statistical support for the association between positive selection and IDRs in proteins.

Fourthly, we have used alternative approaches to study the possibility that the increased frequency of positively selected residues in IDRs is the result of reduced negative selection in these regions due to the fact that they might be less important for protein function. This hypothesis would fit well with preconceptions about protein structure that have stated that structured conformation correlates with functional significance. Most importantly, however, this explanation of our results is contradicted by our data, since the frequency of negatively selected codons is not significantly reduced in IDRs relative to protein regions with a structured conformation. Consistently, other recent reports also suggest that IDRs are under negative selection at a level that may even exceed the level for secondary structure elements [31,32]. To further address the issue, we used the Limacs method to independently predict the relative frequency of functionally import amino acid residues in IDRs in relation to regions of structured conformation. The results are consistent with our codon selection data and show that the predicted frequency of functionally important residues is similar in IDRs and regions with structured conformation.

Other approaches to assess the robustness of our conclusion that the IDRs in proteins are particularly susceptible to positive selection are to test whether protein classes predicted to have high adaptive potential make biological sense, whether they are generally characterized by proteins with high IDR content, as well as whether such proteins are associated with molecular mechanisms that could explain their higher adaptive potential. To test whether particular protein categories are enriched in proteins predicted to be under positive or negative selection, we used the FunCat and ProteinCat catalogues of yeast proteins. Several FunCat categories showed significant over-representation of proteins predicted to be under positive selection. These included functions associated with development, mating, and morphogenesis that contain known targets for adaptive selection. Other categories have to do with virulence, defense and cell signaling as well as many categories related to DNA functions, including transcription. Most of these categories contain many proteins that are potentially relevant targets for adaptive mutation. Fewer FunCat categories showed significant evidence of negative selection but these include classes containing highly conserved proteins involved in detoxification, fermentation and protein folding. The ProteinCat categories that are significantly enriched in proteins predicted to be under negative or positive selection also make sense. Most of the categories under negative selection contain enzymes, which are known to be high in structured regions under negative selection. The three categories enriched in proteins predicted to be under positive selection are all categories containing transcription factors. Individual transcription factors have been suggested to be under positive selection previously [37-39]. Furthermore, transcription factors have been shown to evolve faster than other protein classes in yeast [40]. As predicted by our model, FunCat and ProteinCat categories that are over-represented in proteins predicted to be under positive selection also have a high average IDR content while the reverse is true for categories associated with negative selection.

Our data suggest that the conformational flexibility of IDRs, which might potentially translate to a functional flexibility, could represent a generally evolvable characteristic. IDRs might represent a conformational ground state that provides proteins with an intrinsic ability to adapt new functionality. According to this view, protein regions with regular structure would tend to favor structural and functional specialization but at a cost in terms of evolvability. Consistent with this, experimental studies suggest that naturally occurring proteins are not maximally stable, but rather that they seem to exhibit the minimal level of stability necessary for the environment in which they function [41]. Furthermore, in silico studies have shown that strong selection for structural stability would be expected to lead to reduced evolution of novel protein functions $[42,43]$.

A key question is thus whether there is evidence to support a link between the conformational flexibility of IDRs and functional flexibility? The widespread involvement of IDRs in interactions between protein partners involved in a diverse range of biological functions provides such evidence [44]. Further, IDRs have, as predicted, been shown to adopt different conformations upon interaction with different binding partners [21]. It has long been recognized that alterations affecting gene regulation provide a powerful opportunity for evolution of phenotypic differences between organisms and hence for the adaptation of organisms to new environments [45]. Much attention has focused on studies of adaptive changes that affect the sequence of cis-acting regulatory elements in gene promoters, enhancers or silencers $[46,47]$. However, recent studies have suggested that mutations in trans-acting components, including transcription factors and co-regulators, are also important for the evolutionary adaptation of transcription networks [48-50]. Protein interactions involving transcriptional components have been suggested to play a role in such evolutionary processes $[46,51]$. Several studies have shown evidence of adaptive changes in the protein interaction domains of transcription factor proteins [37-39] and previous computational studies have independently 
shown that transcription factors have a high IDR content $[20,52]$. Interestingly, transcription factor activation domains have been shown to be IDRs $[53,54]$ that interact with other proteins by two-step target-assisted folding mechanisms in which their intrinsically unstructured nature plays an important role $[55,56]$.

\section{Conclusions}

Taken together with previous knowledge, our results thus provide strong evidence for the involvement of IDRs in evolutionary adaptation. Such IDRs are sometimes associated with transcription factors, where they have been relatively well studied, but it is likely that IDRs involved in adaptation may be found in a much broader range of protein classes. The genome-wide nature of the study suggests that the conclusions are significant to most if not all of the proteome. The adaptive nature of IDRs gives a new perspective for understanding the potential adaptive significance of gene variants that arise in nature and medicine.

\section{Materials and methods}

\section{Retrieval of genomic sequences and polymorphism} information

Plain text files containing the $S$. cerevisiae and S. paradoxus reference genome sequences, genomic coordinates of identified SNPs in each of the sequenced isolates (37 S. cerevisiae strains and 27 S. paradoxus strains), and genomic coordinates of the protein coding genes in $S$. cerevisiae were downloaded on 1 September 2007 from the Sanger ftp site [57]. The strains studied are listed in Additional file 1. Only confirmed polymorphisms were used in the subsequent analyses. Details of synonymous and non-synonymous SNPs in S. cerevisiae and S. paradoxus strains are described per protein-coding sequence and per chromosome in Additional files 2, 3, 4, 5, 6 and 7 .

\section{Retrieval of protein coding genes in S. cerevisiae and S. paradoxus}

The coding sequences of all annotated S. cerevisiae protein coding genes in the Saccharomyces Genome Database [58] were extracted from the reference genome sequence, and reverse complemented for genes where transcription occurs from the lower strand. For S. paradoxus, we retrieved the genomic coordinates of genes inferred previously based on synteny and sequence similarity of predicted ORFs in the $S$. paradoxus genome to annotated genes in the $S$. cerevisiae genome [28]. The corresponding coding sequences were extracted from the $S$. paradoxus reference genome sequence and tested for their coding potential in six possible ORFs using the sixpack method of the EMBOSS sequence analysis package [59]. When necessary, the coding sequence was reverse complemented and/or shifted to yield a translatable ORF. No mitochondrial genes were included in the analysis.

\section{Alignment of orthologous genes}

For each $S$. cerevisiae gene where a $S$. paradoxus orthologue could be inferred, a multiple sequence alignment was created consisting of all strain orthologues for which at least one sequence contained at least one SNP relative to the respective reference genome sequence. To assure that the alignment did not result in any frameshifts, translation-assisted alignments were created using DIALIGN-T [60]. To ensure that subsequent analyses were not affected by the occurrence of uncertain alignments, orthologous protein coding gene alignments were filtered at different stringency thresholds based on the level of sequence identity in the alignments of the translated sequences and alignments below the filtering threshold were removed (60\%, 4,029 alignments; $70 \%$, 4,001 alignments; 80\%, 3,198 alignments). Subsequent analyses were performed on each of the three datasets to determine whether the choice of filtering threshold altered the conclusions drawn from subsequent analyses. The choice of filtering criteria did not significantly influence subsequent analysis and results using the alignments with $\geq 70 \%$ are shown in the paper. Details of the DNA sequence alignments used in the study are available on request. The methods used to detect selection assume that all sites in each gene share the same phylogeny [61] and therefore alignments where recombination events were predicted by the GENECONV method [62] using the ' $r$ ' option (only silent sites analyzed), and calculating global $P$-values based on Bonferroni-corrected Karlin-Altschul $P$-values were removed from the subsequent analysis.

\section{Prediction of structured and intrinsically disordered protein regions}

The PSIPRED [63] and VSL2 [64] methods were used to predict the occurrence of structured and disordered protein regions, respectively, in all S. cerevisiae proteins. The protein-coding DNA sequences used as well as the protein sequences translated from them are provided in Additional files 8 and 9, respectively. The VSL2 method was among the best performing in the CASP7 assessment of IDR prediction algorithms [65], and performs particularly well in predicting short disordered regions [64]. Both methods rely on evolutionary information derived from PSI-BLAST generated profiles. For the PSI-BLAST searches we used a filtered version of the Uniref90 database (release 12.8) where transmembrane regions, coiled-coil regions and low-complexity regions had been removed using the pfilt program [66]. Each PSI-BLAST search was performed for three iterations, 
with an E-value threshold of 0.001 for inclusion in a multi-pass model, against the reduced Uniref90 database. A position-specific scoring matrix was produced (option -Q) and this was used as input to the PSIPRED and VSL2 algorithms. Amino acid residues predicted by PSIPRED to belong to state 'helix' or 'extended beta' with a confidence value equal to or larger than 8 were chosen for subsequent analysis of regular secondary structures (Additional files 10 and 11). For prediction of disordered residues, we used both a strict confidence value threshold of 0.8 and a liberal threshold value of 0.5 (Additional files 12 and 13). Any residue sites for which predictions of disordered and regular structure overlapped were removed. Except where stated, the strict confidence value (0.8) was used for IDR prediction in the data shown in the paper. The mean fraction of residues reliably predicted to be in $\alpha$-helical, $\beta$-strand, and intrinsically disordered conformation was $26 \%, 6 \%$ and $23 \%$, respectively. Since the sequences studied using these selection criteria represent only $55 \%$ of amino acid residues, all analyses were also performed using the liberal confidence threshold (0.5) for IDR detection (44\% of residues identified as IDR, $76 \%$ of residues included in analyses). None of the overall conclusions were affected by use of reduced-stringency IDR prediction criteria (0.5). We obtained 1,191 protein regions mapping to known structured domains in the protein database (PDB) and corresponding to 643 yeast proteins from the PFAM database (version 25.0) [67].

\section{Phylogenetic test for selection}

Amino acid residues under selection in inter-strain/species alignments were identified using a codon-based maximum likelihood method implemented in the HyPhy software package [68]. Each alignment was analyzed separately for codon sites under selection using the FEL method. A neighbor-joining tree was built for each alignment under the Tajima-Nei model of nucleotide substitution [69], and the tree topology along with the alignment were used as input. The HKY85 model of nucleotide substitution was used [70]. In the FEL method, a likelihood ratio test is performed to estimate the rates of synonymous $(\alpha)$ and non-synonymous $(\beta)$ substitution at each codon site. If the synonymous substitution rate is higher than the non-synonymous rate $(\alpha$ $>\beta$ ), this is indicative of negative selection, whereas a higher non-synonymous substitution rate $(\beta>\alpha)$ indicates the action of positive selection. The default threshold value of $P \leq 0.1$ was used to reject the nullhypothesis of $\alpha=\beta$ at a codon site.

\section{Population genetic test for selection}

To detect genes under selection, the multiple sequence alignments of all orthologous protein coding genes were subjected to the McDonald-Kreitman test [71] as implemented in the MKtest program of the libsequence package [72]. This test investigates the correlation of polymorphisms within species and their divergence between species, and also distinguishes between synonymous and non-synonymous sites. In a sequence having no evolutionary constraints, the ratio of non-synonymous and synonymous sites that are fixed between species $(\mathrm{dN} / \mathrm{dS})$ should be roughly equal to the ratio of non-synonymous and synonymous sites that are polymorphic within a species (pN/pS), according to the neutral theory of evolution [73]. We refer to the ratio (dN/ $\mathrm{dS}) /(\mathrm{pN} / \mathrm{pS})$ as the fixation index (FI). When negative selection is acting on a locus, non-synonymous mutations are unlikely to become fixed, although they might still contribute to polymorphism within a species. Thus, the ratio $(\mathrm{dN} / \mathrm{dS})$ is expected to be lower than the ratio $(\mathrm{pN} / \mathrm{pS})$, yielding an $\mathrm{FI}<1$. However, if positive selection is acting on a locus, non-synonymous mutations are expected to spread rapidly through the population, thus having a greater effect on divergence than on polymorphism. In this case, the ratio $(\mathrm{dN} / \mathrm{dS})$ is expected to be higher than the ratio $(\mathrm{pN} / \mathrm{pS})$, yielding an $\mathrm{FI}>1$. We calculated the FI for each gene and performed a Fisher's exact test of the null hypothesis of independence between the two ratios $(\mathrm{dN} / \mathrm{dS})$ and $(\mathrm{pN} / \mathrm{pS})$. Rejection of the null hypothesis at the $5 \%$ significance level was taken as an indication of either negative or positive selection, depending on the FI value (Additional file 14). The occurrence of slightly deleterious mutations is known to cause an underestimation of the level of adaptive evolution [74], and a frequently used approach to control for some of the effects of these mutations is to remove polymorphisms segregating at low frequencies. Thus, all SNPs occurring in less than 15\% of the strains within a species were removed, as this has been demonstrated to be an appropriate threshold [75]. Additionally, average proteome McDonald-Kreitman tests were performed by merging all aligned codons encoding amino acid residues reliably predicted to be in regions of $\alpha$ helical, $\beta$-sheet or intrinsically disordered conformation, and performing the calculations described above on each of the three resulting composite alignments (Additional file 15). The $(\mathrm{G}+\mathrm{C})$ content of each $S$. cerevisiae gene was calculated using the geecee program of the EMBOSS package.

\section{Prediction and analysis of functionally important amino acid residues}

We applied the Limacs method [76,77] to predict the occurrence of functionally important sites in the amino acid sequence of translated S. cerevisiae genes. Given a multiple sequence alignment, Limacs uses a template library for prediction of functionally important sites in the 
alignment. Since the method is based on known functional sites in conserved functional domains [77], we constrained the analysis to mapped Pfam domains [78]. All annotated Pfam domains were mapped to the translated yeast genes using reverse position-specific BLAST (RPS-BLAST). Domains that mapped to at least one of the yeast proteins were subjected to analysis by Limacs to predict the location of functional sites. To score positive as a functional site, sites were required to have a query column versus template pattern score (QT score) of at least 0.95 , a QT Zscore of at least 1 , and randomization scores QRn and TRn lower than 0.01. The distribution of predicted functional sites in mapped Pfam domains was analyzed for residues in regions of intrinsically disordered and regular conformation, and differences in the distribution were assessed by a $\chi^{2}$ test, where a $2 \times 2$ contingency table of functional sites in IDRs (LI) and non-IDRs (LnI), and of non-functional sites in IDRs (nLI) and in non-IDRs (nLnI) was built. The Limacs functional sites index (LI/nLI)/(LnI/ $\mathrm{nLnI}$ ) was used to indicate whether there was a relative abundance (index above one) or depletion (index below one) of predicted functional sites in IDRs compared to non-IDRs. Predicted functional sites in each gene are listed in Additional file 16. To ensure the robustness of the obtained results, the comparison was repeated using various IDR prediction reliability cutoff values. Furthermore, to avoid bias from over-represented domains, a conservative filtering procedure was also applied, in which only one of the mapped protein regions was analyzed in cases where a domain mapped to more than one protein.

\section{Assessment of differences in degree of selection between protein regions of regular and intrinsically disordered structure}

Spearman's rank correlation coefficient $\left(r_{S}\right)$ was calculated to assess the correlation between secondary structure content and FI in genes where the McDonaldKreitman test rejected the null hypothesis of neutral evolution. In the same manner, the correlation was assessed between $(\mathrm{G}+\mathrm{C})$ content and secondary structure content, and between $(\mathrm{G}+\mathrm{C})$ content and FI. Because of the large sample size $(n)$, a normal distribution was assumed and the statistical significance was determined by calculating $\mathrm{z}=\mathrm{r}_{\mathrm{S}} \sqrt{ } \mathrm{n}-1$.

The intra-genic correlation between secondary structure content and FI was assessed for each gene by a sliding-window analysis, where structure content and FI were calculated within non-overlapping windows of size 25 codons for all aligned orthologous genes. Spearman's rank correlation coefficient was calculated for the resulting data points, indicating the correlation of structure content and FI for each individual gene. Only informative windows were analyzed, that is, regions for which a FI value could be calculated. If the number of informative windows from a gene alignment was more than 30, a normal distribution was assumed and an approximate $\mathrm{z}$-value was calculated as above. In cases where the number of informative windows was fewer than 30 but more than 5, significance was assessed by consulting a table of pre-calculated critical $r_{S}$ values. If the analysis produced five or fewer informative windows, no correlation analysis was performed on the gene.

The distribution of codon sites predicted to be under positive or negative selection was likewise assessed between the IDR, $\alpha$-helix and $\beta$-sheet conformational states. A series of $2 \times 2$ contingency tables was generated describing the frequencies of two types of codon sites in two different structure states, and for each table the difference in distribution was assessed by a $\chi^{2}$ test. To independently investigate whether an observed difference in the distribution of a given type of codon in a certain conformational state could be expected by chance, a randomization procedure was applied. The number of codon sites, $n$, of a given type $C$ in the investigated structure state $S$ was counted and summed over all genes, as was the total number of codon sites, $N$, in conformational state $S$. The randomization test entailed performing 10,000 re-samples, where $N$ codon sites were randomly chosen from any gene, and counting the resulting number of sites, $n$ ', of type $C$. The observed number of type $C$ sites, $n$, in intrinsically disordered regions was then compared to the simulated distribution of $n$ 'values, and the null hypothesis of equal distribution in disordered regions and regions with secondary structure was rejected by a two-tailed $t$-test $(P \leq 0.001)$.

The intra-genic correlation between occurrence of type $C$ sites and secondary structure content was assessed for each gene by a sliding-window analysis, where non-overlapping windows of size 25 codons were moved over the gene, counting the number of type $C$ sites and calculating the secondary structure content in each window. Spearman's rank correlation coefficient was calculated to assess the correlation between secondary structure content and type $C$ codon site distribution, and the statistical significance was estimated as described above, discarding windows with no type $C$ codons.

\section{Assessment of differences in degree of selection between functional categories of proteins}

We adopted the MIPS FunCat and ProteinCat annotation schemes [34] to assign each gene into one or more functional categories. Excess or depletion of codon sites under positive selection in a given functional category was assessed by a randomization test. In a category with a sum of $N$ codon sites over all constituent genes, the observed number of sites under selection, $n$, was compared to the empirical distribution of the number of selected sites, which was derived by choosing $N$ codon sites from random genes in any functional category and 
counting the number of sites under selection, $n$, then repeating this process 10,000 times. A two-tailed $t$-test was performed to estimate if the observed number of sites under positive selection in a certain category deviated significantly from the random expectation.

\section{Additional material}

Additional file 1: Yeast strain isolates used in the study

Additional file 2: Non-synonymous SNPs in S. cerevisiae genes studied. The nature of each amino acid change for each changed amino acid in each strain is shown for each of 3,639 genes.

Additional file 3: Non-synonymous SNPs in S. paradoxus genes studied. The nature of each amino acid change for each changed amino acid in each strain is shown for each of 3,691 genes.

Additional file 4: Synonymous SNPs in S. cerevisiae genes studied. The identity of each affected amino acid in each affected strain is shown for each of 3,737 genes.

Additional file 5: Synonymous SNPs in S. paradoxus genes studied The identity of each affected amino acid in each affected strain is shown for each of 3,756 genes.

Additional file 6: Chromosomal location of protein-coding region SNPs in the different strains of $S$. cerevisiae. The direction of each gene as well as the base change in each SNP and its affect on codons and encoded amino acids is shown.

Additional file 7: Chromosomal location of protein-coding region SNPs in the different strains of S. paradoxus. The direction of each gene as well as the base change in each SNP and its affect on codons and encoded amino acids is shown.

Additional file 8: FastA file containing the ORF DNA sequence of all analyzed S. cerevisiae genes. Strand, phase, chromosone and genomic start/finish coordinates are specified in the header line.

Additional file 9: FastA file containing the protein sequence translated from the ORFs of all analyzed $S$. cerevisiae genes.

Additional file 10: Fraction of amino acid residues for each protein that are predicted by the PSIPRED method to adopt $\alpha$-helical conformation, using a confidence value threshold of 8 .

Additional file 11: Fraction of amino acid residues for each protein that are predicted by the PSIPRED method to adopt $\beta$-sheet conformation, using a confidence value threshold of 8

Additional file 12: Fraction of amino acid residues for each protein that are predicted by the VSL2 method to adopt intrinsically disordered conformation, using a confidence value threshold of 0.8 Additional file 13: Fraction of amino acid residues for each protein that are predicted by the VSL2 method to adopt intrinsically disordered conformation, using a confidence value threshold of 0.5 . Additional file 14: Fixation index of each investigated protein coding gene along with the associated $P$-value. The $\mathrm{Fl}$ was calculated using the MKtest program and is defined as the ratio (dN/dS)/(pN/pS).

Additional file 15: Fixation index calculated for the merged aligned regions in $\alpha$-helical, $\beta$-strand, or intrinsically disordered conformation from all analyzed proteins, with or without removal of genes with a fixation index deviating more than three standard deviations from the mean of the entire data set.

Additional file 16: Amino acid positions of functional sites in each investigated protein as predicted by the Limacs method for known pfam domains mapped to the proteins.

\section{Abbreviations}

FEL: fixed effects likelihood; Fl: fixation index (calculated in the McDonaldKreitman test); IDR: intrinsically disordered region; MIPS: Munich Information
Center for Protein Sequences; ORF: open reading frame; SNP: single nucleotide polymorphism

\section{Acknowledgements}

The authors thank colleagues at Södertörn University and the Karolinska Institute for helpful discussions. The work was supported by a grant from the Baltic Sea Foundation and AW is also supported by grants from the Swedish Research Council and the Swedish Cancer Society.

\section{Author details}

${ }^{1}$ School of Life Sciences, Södertörn University, SE-141 89 Huddinge, Sweden. ${ }^{2}$ Clinical Research Center, Novum Level 5, Department of Laboratory Medicine and Center for Biosciences, Karolinska Institutet, SE-141 86 Huddinge, Sweden.

\section{Authors' contributions}

$\mathrm{JN}$ was involved in the conception and planning of the study, carried out the bioinformatic studies and was involved in interpretation of results and drafting the manuscript. MG was involved in the conception and planning of the study as well as interpretation of results and preparation of the manuscript. AW was involved in the conception and planning of the study as well as interpretation of results and writing the manuscript. All authors read and approved the final manuscript.

\section{Competing interests}

The authors declare that they have no competing interests.

Received: 26 January 2011 Revised: 31 May 2011

Accepted: 19 July 2011 Published: 19 July 2011

\section{References}

1. Kimura M: Evolutionary rate at the molecular level. Nature 1968 217:624-626

2. MacCallum C, Hill E: Being positive about selection. PLoS Biol 2006, 4:e87

3. Sabeti P, Reich D, Higgins J, Levine H, Richter D, Schaffner S, Gabriel S, Platko J, Patterson N, McDonald G, Ackerman H, Campbell S, Altshuler D, Cooper R, Kwiatkowski D, Ward R, Lander E: Detecting recent positive selection in the human genome from haplotype structure. Nature 2002, 419:832-837.

4. Tang K, Thornton $\mathrm{K}$, Stoneking M: A new approach for using genome scans to detect recent positive selection in the human genome. PLOS Biol 2007, 5:e171.

5. Yang Z: PAML 4: phylogenetic analysis by maximum likelihood. Mol Biol Evol 2007, 24:1586-1591.

6. Chen S, Hung C, Xu J, Reigstad C, Magrini V, Sabo A, Blasiar D, Bieri T, Meyer R, Ozersky P, Armstrong J, Fulton R, Latreille J, Spieth J, Hooton T, Mardis E, Hultgren S, Gordon J: Identification of genes subject to positive selection in uropathogenic strains of Escherichia coli: a comparative genomics approach. Proc Natl Acad Sci USA 2006, 103:5977-5982.

7. Nielsen R, Bustamante C, Clark A, Glanowski S, Sackton T, Hubisz M, FledelAlon A, Tanenbaum D, Civello D, White T, Sninsky J, Adams M, Cargill M: A scan for positively selected genes in the genomes of humans and chimpanzees. PLOS Biol 2005, 3:e170.

8. Petersen L, Bollback J, Dimmic M, Hubisz M, Nielsen R: Genes under positive selection in Escherichia coli. Genome Res 2007. 17:1336-1343.

9. Ramensky V, Bork P, Sunyaev S: Human non-synonymous SNPs: server and survey. Nucleic Acids Res 2002, 30:3894-3900.

10. The International HapMap Consortium: The International HapMap Project. Nature 2003, 426:789-796.

11. Kirschner M, Gerhart J: Evolvability. Proc Natl Acad Sci USA 1998, 95:8420-8427.

12. Pigliucci M: Is evolvability evolvable?. Nat Rev Genet 2008, 9:75-82.

13. Pigliucci M: Do we need an extended evolutionary synthesis?. Evol Int $J$ Org Evol 2007, 61:2743-2749.

14. Cowen L, Lindquist S: Hsp90 potentiates the rapid evolution of new traits: drug resistance in diverse fungi. Science 2005, 309:2185-2189.

15. Parter M, Kashtan N, Alon U: Facilitated variation: how evolution learns from past environments to generalize to new environments. PLOS Comput Biol 2008, 4:e1000206.

16. Wagner A: Robustness, evolvability, and neutrality. FEBS Lett 2005, 579:1772-1778. 
17. Wagner A: Robustness and evolvability: a paradox resolved. Proc Bio/ SC 2008, 275:91-100.

18. Dunker A, Oldfield C, Meng J, Romero P, Yang J, Chen J, Vacic V, Obradovic Z, Uversky V: The unfoldomics decade: an update on intrinsically disordered proteins. BMC Genomics 2008, 9(Suppl 2):S1.

19. Fink A: Natively unfolded proteins. Curr Opin Struct Biol 2005, 15:35-41.

20. Lobley A, Swindells M, Orengo C, Jones D: Inferring function using patterns of native disorder in proteins. PLoS Comput Biol 2007, 3:e162.

21. Wright PE, Dyson HJ: Linking folding and binding. Curr Opin Struct Biol 2009, 19:31-38

22. Tompa P, Dosztanyi Z, Simon I: Prevalent structural disorder in E. coli and S. cerevisiae proteomes. J Proteome Res 2006, 5:1996-2000.

23. Bogatyreva NS, Finkelstein AV, Galzitskaya OV: Trend of amino acid composition of proteins of different taxa. J Bioinformatics Comput Biol 2006, 4:597-608.

24. Goffeau A, Barrell B, Bussey H, Davis R, Dujon B, Feldmann H, Galibert F, Hoheisel J, Jacq C, Johnston M, Louis E, Mewes H, Murakami Y, Philippsen P, Tettelin H, Oliver S: Life with 6000 genes. Science 1996, 274:546.

25. Ghaemmaghami S, Huh W, Bower K, Howson R, Belle A, Dephoure N, O'Shea E, Weissman J: Global analysis of protein expression in yeast. Nature 2003, 425:737-741.

26. Cliften P, Sudarsanam P, Desikan A, Fulton L, Fulton B, Majors J, Waterston $\mathrm{R}$, Cohen $\mathrm{B}$, Johnston M: Finding functional features in Saccharomyces genomes by phylogenetic footprinting. Science 2003, 301:71-76

27. Dujon B, Sherman D, Fischer G, Durrens P, Casaregola S, Lafontaine I, Montigny JD, Marck C, Neuvéglise C, Talla E, Goffard N, Frangeul L, Aigle M, Anthouard V, Babour A, Barbe V, Barnay S, Blanchin S, Beckerich J, Beyne E, Bleykasten C, Boisramé A, Boyer J, Cattolico L, Confanioleri F, Daruvar AD, Despons L, Fabre E, Fairhead C, Ferry-Dumazet H, et al: Genome evolution in yeasts. Nature 2004, 430:35-44.

28. Kellis M, Patterson N, Endrizzi M, Birren B, Lander E: Sequencing and comparison of yeast species to identify genes and regulatory elements. Nature 2003, 423:241-254.

29. Rossignol T, Lechat P, Cuomo C, Zeng Q, Moszer I, d'Enfert C: CandidaDB: a multi-genome database for Candida species and related Saccharomycotina. Nucleic Acids Res 2008, , 36 Database: D557-561.

30. Liti G, Carter D, Moses A, Warringer J, Parts L, James S, Davey R, Roberts I, Burt A, Koufopanou V, Tsai I, Bergman C, Bensasson D, O'Kelly M, Oudenaarden Av, Barton D, Bailes E, Nguyen A, Jones M, Quail M, Goodhead I, Sims S, Smith F, Blomberg A, Durbin R, Louis E: Population genomics of domestic and wild yeasts. Nature 2009, 458:337-341.

31. Schaefer $C$, Schlessinger A, Rost B: Protein secondary structure appears to be robust under in silico evolution while protein disorder appears not to be. Bioinformatics 2010, 26:625-631.

32. Tompa P, Kalmar L: Power law distribution defines structural disorder as a structural element directly linked with function. J Mol Biol 2010, 403:346-350.

33. Chen J, Romero P, Uversky V, Dunker A: Conservation of intrinsic disorder in protein domains and families: I. A database of conserved predicted disordered regions. J Proteome Res 2006, 5:879-887.

34. Ruepp A, Zollner A, Maier D, Albermann K, Hani J, Mokrejs M, Güldener ITU, Mannhaupt G, Münsterkötter M, Mewes H: The FunCat, a functional annotation scheme for systematic classification of proteins from whole genomes. Nucleic Acids Res 2004, 32:5539-5545.

35. Mayrose I, Doron-Faigenboim A, Bacharach E, Pupko T: Towards realistic codon models: among site variability and dependency of synonymous and non-synonymous rates. Bioinformatics 2007, 23:i319-327.

36. Nozawa M, Suzuki Y, Nei M: Reliabilities of identifying positive selection by the branch-site and the site-prediction methods. Proc Natl Acad Sci USA 2009, 106:6700-6705.

37. Luo C, Lu X, Stubbs L, Kim J: Rapid evolution of a recently retroposed transcription factor YY2 in mammalian genomes. Genomics 2006, 87:348-355.

38. Maiti S, Doskow J, Sutton K, Nhim R, Lawlor D, Levan K, Lindsey J, Wilkinson M: The Pem homeobox gene: rapid evolution of the homeodomain, $\mathrm{X}$ chromosomal localization, and expression in reproductive tissue. Genomics 1996, 34:304-316.
39. Zhang J, Webb D, Podlaha O: Accelerated protein evolution and origins of human-specific features: Foxp2 as an example. Genetics 2002, 162:1825-1835

40. Beskow A, Wright AP: Comparative analysis of regulatory transcription factors in Schizosaccharomyces pombe and budding yeasts. Yeast 2006, 23:929-935.

41. Arnold F, Wintrode P, Miyazaki K, Gershenson A: How enzymes adapt: lessons from directed evolution. Trends Biochem Sci 2001, 26:100-106.

42. Bloom J, Wilke C, Arnold F, Adami C: Stability and the evolvability of function in a model protein. Biophys J 2004, 86:2758-2764.

43. Basu M, Carmel L, Rogozin I, Koonin E: Evolution of protein domain promiscuity in eukaryotes. Genome Res 2008, 18:449-461.

44. Shimizu K, Toh $\mathrm{H}$ : Interaction between intrinsically disordered proteins frequently occurs in a human protein-protein interaction network. J Mol Biol 2009, 392:1253-1265

45. King M, Wilson A: Evolution at two levels in humans and chimpanzees. Science 1975, 188:107-116.

46. Hsia C, McGinnis W: Evolution of transcription factor function. Curr Opin Genet Dev 2003, 13:199-206.

47. Tirosh I, Barkai N, Verstrepen KJ: Promoter architecture and the evolvability of gene expression. J Biol 2009, 8:95.

48. Choi JK, Kim YJ: Epigenetic regulation and the variability of gene expression. Nat Genet 2008, 40:141-147.

49. Tirosh I, Reikhav S, Levy AA, Barkai N: A yeast hybrid provides insight into the evolution of gene expression regulation. Science 2009, 324:659-662.

50. Wittkopp PJ, Haerum BK, Clark AG: Evolutionary changes in cis and trans gene regulation. Nature 2004, 430:85-88.

51. Lynch $V$, Wagner $G$ : Resurrecting the role of transcription factor change in developmental evolution. Evolution 2008, 62:2131-2154.

52. Liu J, Narayanan B, Oldfield C, Su E, Uversky V, Dunker A: Intrinsic disorder in transcription factors. Biochemistry 2006, 45:6873-6888.

53. McEwan IJ, Dahlman-Wright K, Ford J, Wright AP: Functional interaction of the c-Myc transactivation domain with the TATA binding protein: evidence for an induced fit model of transactivation domain folding. Biochemistry 1996, 35:9584-9593.

54. Radhakrishnan I, Perez-Alvarado GC, Parker D, Dyson HJ, Montminy MR, Wright PE: Solution structure of the KIX domain of CBP bound to the transactivation domain of CREB: a model for activator:coactivator interactions. Cell 1997, 91:741-752.

55. Ferreira ME, Hermann S, Prochasson P, Workman JL, Berndt KD, Wright AP: Mechanism of transcription factor recruitment by acidic activators. J Biol Chem 2005, 280:21779-21784

56. Hermann S, Berndt KD, Wright AP: How transcriptional activators bind target proteins. J Biol Chem 2001, 276:40127-40132.

57. Sanger FTP.. [ftp://ftp.sanger.ac.uk/pub/dmc/yeast].

58. Saccharomyces Genome Database.. [http://www.yeastgenome.org].

59. Rice P, Longden I, Bleasby A: EMBOSS: the European Molecular Biology Open Software Suite. Trends Genet 2000, 16:276-277.

60. Subramanian A, Weyer-Menkhoff J, Kaufmann M, Morgenstern B: DIALIGN$\mathrm{T}$ : an improved algorithm for segment-based multiple sequence alignment. BMC Bioinformatics 2005, 6:66.

61. Anisimova M, Nielsen R, Yang Z: Effect of recombination on the accuracy of the likelihood method for detecting positive selection at amino acid sites. Genetics 2003, 164:1229-1236.

62. Sawyer S: Statistical tests for detecting gene conversion. Mol Biol Evol 1989, 6:526-538.

63. Jones D: Protein secondary structure prediction based on positionspecific scoring matrices. J Mol Biol 1999, 292:195-202.

64. Peng K, Radivojac P, Vucetic S, Dunker A, Obradovic Z: Length-dependent prediction of protein intrinsic disorder. BMC Bioinformatics 2006, 7:208

65. Bordoli $L$, Kiefer F, Schwede T: Assessment of disorder predictions in CASP7. Proteins 2007, 69(Suppl 8):129-136.

66. Jones $\mathrm{D}$, Taylor $\mathrm{W}$, Thornton J: A model recognition approach to the prediction of all-helical membrane protein structure and topology. Biochemistry 1994, 33:3038-3049.

67. PFAM database.. [ftp://ftp.sanger.ac.uk//pub/databases/Pfam//releases/ Pfam25.0/pdbmap.gz].

68. Pond S, Frost $S$, Muse S: HyPhy: hypothesis testing using phylogenies. Bioinformatics 2005, 21:676-679. 
69. Tajima F, Nei M: Estimation of evolutionary distance between nucleotide sequences. Mol Biol Evol 1984, 1:269-285.

70. Hasegawa M, Kishino H, Yano T: Dating of the human-ape splitting by a molecular clock of mitochondrial DNA. J Mol Evol 1985, 22:160-174.

71. McDonald J, Kreitman M: Adaptive protein evolution at the Adh locus in Drosophila. Nature 1991, 351:652-654.

72. Thornton T: Libsequence: a C++ class library for evolutionary genetic analysis. Bioinformatics 2003, 19:2325-2327.

73. Kimura M: The Neutral Theory of Molecular Evolution Cambridge: Cambridge Univeristy Press; 1983.

74. Fay J, Wycoff G, Wu C-I: Positive and negative selection on the human genome. Genetics 2001, 158:1227-1234.

75. Charlesworth J, Eyre-Walker A: The McDonald-Kreitman test and slightly deleterious mutations. Mol Biol Evol 2008, 25:1007-1015.

76. Chakrabarti S, Lanczycki C: Analysis and prediction of functionally important sites in proteins. Protein Sci 2007, 16:4-13.

77. Lanczycki C, Chakrabarti S: A tool for the prediction of functionally important sites in proteins using a library of functional templates. Bioinformation 2008, 2:279-283.

78. Finn R, Tate J, Mistry J, Coggill P, Sammut S, Hotz H, Ceric G, Forslund K, Eddy S, Sonnhammer E, Bateman A: The Pfam protein families database. Nucleic Acids Res 2008, 36:D281-D288.

doi:10.1186/gb-2011-12-7-r65

Cite this article as: Nilsson et al:: Proteome-wide evidence for enhanced positive Darwinian selection within intrinsically disordered regions in proteins. Genome Biology 2011 12:R65.

\section{Submit your next manuscript to BioMed Central and take full advantage of:}

- Convenient online submission

- Thorough peer review

- No space constraints or color figure charges

- Immediate publication on acceptance

- Inclusion in PubMed, CAS, Scopus and Google Scholar

- Research which is freely available for redistribution

Submit your manuscript at www.biomedcentral.com/submit 